\title{
ICMI Column - Espace Mathématique Francophone 2018
}

Jean-Luc Dorier (University of Geneva, Switzerland)

Launched by the French Sub-Commission of the ICMI (CFEM - Commission française de l'enseignement mathématique) during the World Mathematical Year 2000, the series of Espace Mathématique Francophone conferences (occurring every three years) is built on the notion of "region" defined in linguistic rather than geographical terms, French being the common language amongst participants. It is recognised as a regional conference of the International Commission on Mathematical Instruction (ICMI) - https://www.mathunion.org/icmi/conferences/ icmi-regional-conferences.
L'Espace Mathématique Francophone (EMF) was set up to promote reflection and exchanges within the French-speaking world on the vital issues of mathematics education in today's societies, at primary, secondary and higher levels, as well as on issues relating to initial and in-service teacher training. The EMF contributes to the development of a Francophone community rich in cultural diversity around mathematics education at the crossroads of continents, cultures and generations.

All information about the EMF, including all proceedings (with an internal browser), the composition of 
the executive bureau and the status of the conference can be found at www.emf.unige.ch, where you can also subscribe to the distribution list.

The seventh EMF (EMF-2018) will be held in Paris (France):

Mathematics on stage, bridges between disciplines

Paris, 22 to 26 October 2018

https://emf2018.sciencesconf.org/

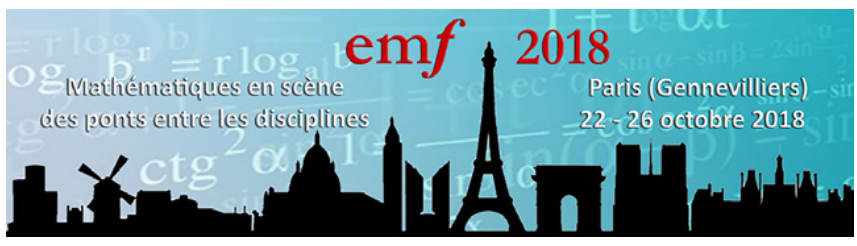

Mathematics has been built and continues to be built in interaction with other disciplines. The historical testimonies that have come down to us show that several mathematical notions were born as answers to problems (concrete needs of individuals or groups) and that they evolved in a dynamic of producing tools or concepts and theoretical results. This dynamic was sometimes generated in other fields and sometimes in response to a need for generalisation and theorisation of mathematics itself.

Whilst remaining a means of expressing problems posed by other disciplines and a powerful set of tools for solving some of these problems, mathematics can also be seen as a set of tools for understanding the world and its evolution. It is in the development and use of models to understand or transform this world that mathematical activity tends today to get realised, often in collaboration with other scientific disciplines.

Moreover, the growing presence of technological tools in social, cultural and educational contexts amplifies the complexity of contemporary realities. Understanding the relationship between these tools and the models and algorithms they operationalise is a challenge that must be met if critical thinking and genuine citizen participation are to be exercised. Paradoxically, mathematics has never been so present but with so little visibility.

It becomes essential to understand the complexity of the world in a multidisciplinary, holistic and systematic approach. The educational system does not escape this approach and therefore thinking about mathematics education in relation to other subjects in today's changing world is a way of thinking about the tools needed by today's pupils to live in tomorrow's world.

From an institutional point of view, collaboration between disciplines is increasingly promoted through curriculum reforms in different countries, often as a solution chosen by the institution to help students understand complexity. Thus, various mechanisms promote the convocation of several disciplines: approaches to solving complex problems, investigative approaches, project pedagogy, etc. In addition, in some countries, there are new profiles/competitions for bivalent teachers, in addition to the installation, in some contexts, of multidisci- plinary teaching teams. But do all these reforms imply a real exchange between disciplines or rather a minimal collaboration that is limited to juxtaposition?

The design of genuine multidisciplinary/interdisciplinary learning situations requires significant research work to build authentic bridges between disciplines. In addition, the collaboration of specialists in the various disciplines involved seems necessary, both to ensure the quality and authenticity of the situations proposed and to encourage a significant contribution to learning and avoid the great risk of betraying one or other of the disciplines.

The crucial question of teacher training in mathematics inevitably arises. The challenge here would be to open mathematics teachers to other modes of scientific thought, in particular to their transversal nature, but also to initiate them into epistemological reflection on the articulation of knowledge relating to the different disciplines.

These issues of multidisciplinary and interdisciplinary approaches in the teaching and learning of mathematics are at the heart of the theme of the Espace Mathématique Francophone 2018 conference.

Several questions thus feed this theme:

- To which societal, research and teaching problems does the question of interdisciplinary apply?

- How can the participation of mathematics in interdisciplinary activities be qualified in relation to other disciplines from epistemological and teaching points of view?

- What challenges and opportunities does the articulation of concepts from different disciplines pose for teaching?

- How do teaching practices take into account institutional injunctions?

- What are the contributions to a student's learning and what are the difficulties?

Finally, a fundamental question involves the conditions that are conducive to a "true" collaboration between disciplines, which favours mutual insights between these disciplines. It is all these questions and challenges that the Espace Mathématique Francophone 2018 conference proposes to address through plenary sessions, working groups and special projects. 